\title{
High-level SETD1B gene expression is associated with unfavorable prognosis in hepatocellular carcinoma
}

\author{
DONG CHEN $^{1 *}$, TIELING LI ${ }^{2 *}$, CHENG WANG $^{2}$, GUANGLIN LEI $^{1}$, RUILAN WANG $^{1}$, ZHAOHAI WANG $^{1}$, \\ LINXIANG YU ${ }^{1}$, JIN YAN $^{1}$, PEIRUI ZHANG ${ }^{1}$, XILIANG WANG $^{3}$, SHAOGENG ZHANG $^{1}$ and PENGHUI YANG ${ }^{1,3}$ \\ ${ }^{1}$ Hepatobiliary Department, Beijing 302 Hospital, Beijing 100039; ${ }^{2}$ Department of Cardiology, \\ Chinese People's Liberation Army General Hospital, Beijing 100853; ${ }^{3}$ State Key Laboratory of Pathogens and Biosecurity, \\ Beijing Institute of Microbiology and Epidemiology, Beijing 100071, P.R. China
}

Received April 4,2018; Accepted November 23, 2018

DOI: $10.3892 / \mathrm{mmr} .2019 .9832$

\begin{abstract}
The SET domain-containing 1B (SETD1B) gene is involved in multiple biological processes, including tumor development and progression. However, the role of SETD1B in hepatocellular carcinoma (HCC) is largely unexplored. The present study, examined the expression of SETD1B in patients with HCC and assessed its clinical significance. Reverse transcriptase quantitative polymerase chain reaction and western blot analysis results revealed that the expression levels of SETD1B mRNA and protein were significantly increased in HCC tumor tissues compared with the adjacent normal tissues. In addition, an analysis of the patient clinical factors indicated that increased levels of SETD1B expression were associated with tumor size, clinical stage and liver cirrhosis. Patients with HCC with decreased levels of SETD1B expression exhibited longer survival times compared with those with increased levels of SETD1B expression. In addition, Cox's regression analysis results implied that the upregulation of SETD1B was an independent prognostic marker in patients with HCC. Taken together, the results demonstrated that SETD1B is essential in the progression of HCC and may be used as a potential prognostic marker and therapeutic target in HCC.
\end{abstract}

\section{Introduction}

Hepatocellular carcinoma (HCC) is one of the leading human malignancies prevalent worldwide, and half of the incident cases each year occur in China $(1,2)$. In general, the majority of patients with HCC are diagnosed in the clinic at a late

Correspondence to: Dr Penghui Yang or Dr Shaogeng Zhang, Hepatobiliary Department, Beijing 302 Hospital, 100 West 4th Ring Road, Beijing 100039, P.R. China

E-mail: ypenghuiamms@hotmail.com

E-mail: zhangsg302@hotmail.com

${ }^{*}$ Contributed equally

Key words: hepatocellular carcinoma, SET domain-containing 1B stage of disease progression, and therefore do not have the opportunity for a surgical resection. At present, sorafenib and regorafenib are the only United States of America Food and Drug Administration-approved molecularly targeted drugs for patients with HCC (3). However, the efficacy of HCC treatments remains limited and unsatisfactory. Therefore, to improve the prognosis and quality of life of patients with HCC, novel early diagnostic biomarkers and therapeutic drug targets are urgently required.

SET domain-containing 1B (SETDIB), also known as KMT2G or Set1B, is an important component of the histone methyltransferase complex that generates trimethylated histone $\mathrm{H} 3$ at Lys4 and has been implicated in multiple biological processes (4). The SETDIB gene is located on chromosome 1q12 and encodes a 130-kDa protein with several functional domains. A previous study demonstrated that SETD1B associates with a $450-\mathrm{kDa}$ complex that contains all five noncatalytic components of the SET domain containing 1A (SET1A) complex: CXXC finger protein 1, AT-rich interaction domain 4A, ASH2 like, histone lysine methyltransferase complex subunit, WD repeat domain 5 and WD repeat domain 82 (5). The mixed lineage leukemia $(M L L)$ family of proteins, including MLL1-MLL4, SET1A and SETDIB, specifically methylates Lys4 of histone $\mathrm{H} 3$ and serves a vital role in the transcriptional regulation of genes (6). In our previous study, it was identified that $S E T D 1 B$ was the most frequently mutated gene in primary hepatic neuroendocrine tumor, and that one of the three SETD1B mutants, A1054del, promoted cell proliferation, migration and invasion (7). However, the underlying role of SETD1B in liver carcinogenesis was not addressed. Therefore, an investigation into the expression patterns of SETD1B and its clinical significance in the development and progression of HCC was warranted.

\section{Materials and methods}

Clinical samples. Fresh surgical tumor samples from 76 patients with HCC were collected from the Hepatobiliary Department of Beijing 302 Hospital (Beijing, China) between October 2013 and March 2018 and were examined using reverse transcription quantitative polymerase chain reaction (RT-qPCR) and western blot analysis. For the immunohistochemical (IHC) analysis, 
paraffin-embedded HCC samples were collected between October 2013 and March 2015. The HCC tissues were fixed in $10 \%$ formalin for $24 \mathrm{~h}$ at room temperature, embedded in paraffin and cut into $4-\mu \mathrm{m}$ sections. Within half an hour of isolation, HCC tissues and adjacent tissue samples were quickly placed into liquid nitrogen. Adjacent tissue samples were taken at a distance $>3 \mathrm{~cm}$ from the cancer tissues. Written informed consent was obtained from the enrolled patients with HCC. The present study was approved by The Ethics Committee of Beijing 302 Hospital.

Cell lines and cell culture. The human liver cancer 97L and HCCLM3 cell lines and the normal human liver LO2 cell line used in the present study were obtained from the Experimental Center of Beijing 302 Hospital (Beijing, China). All these cell lines were maintained and in Dulbecco's modified Eagle's medium (Gibco; Thermo Fisher Scientific, Inc., Waltham, MA, USA) supplemented with $10 \%$ fetal bovine serum (Gibco; Thermo Fisher Scientific, Inc.) and incubated at $37^{\circ} \mathrm{C}$ with $5 \% \mathrm{CO}_{2}$.

$R T$ - $q P C R$. Total RNA from the frozen tissue samples of 76 patients with $\mathrm{HCC}$ was extracted using TRIzol ${ }^{\circledR}$ reagent (Thermo Fisher Scientific, Inc.) according to the manufacturer's instructions. SETD1B expression levels were quantified by RT-qPCR methods conducted in an ABI 7500 instrument (Applied Biosystems; Thermo Fisher Scientific, Inc.) using the Maxima SYBR-Green RT-qPCR master mix (Thermo Fisher Scientific, Inc.) according to the protocol of the manufacturer. All experimental samples were normalized to a human GAPDH control. The sequences of the RT-PCR primers were as follows: SETD1B forward, 5'-CTGGGTCTACCATCCCTC CA-3' and reverse, 5'-CTTCCGGAACTTGAGCTGGT-3'; GAPDH forward, 5'-CAGCCTCAAGATCATCAGCA-3' and reverse, 5'-TGTGGTCATGAGTCCTTCCA-3'. The amplification procedure consisted of an initial denaturation at $95^{\circ} \mathrm{C}$ for $5 \mathrm{~min}$, followed by 40 cycles of denaturation at $95^{\circ} \mathrm{C}$ for $15 \mathrm{sec}$, and annealing and extension at $60^{\circ} \mathrm{C}$ for $30 \mathrm{sec}$. The $2^{-\Delta \Delta \mathrm{Cq}}$ method was used to analyze SETD1B expression levels relative to the GAPDH control (8).

Western blot analysis. The total proteins were extracted from surgical samples from the patients with HCC using Tissue Protein Extraction Reagent (Pierce; Thermo Fisher Scientific, Inc.) and quantified using a Bicinchoninic Acid Protein Assay (Pierce; Thermo Fisher Scientific, Inc.). A total of $30 \mu \mathrm{g}$ protein was loaded per lane. Proteins were separated by $12 \%$ SDS-PAGE and were subsequently transferred onto polyvinylidene fluoride (PVDF) membranes (EMD Millipore, Billerica, MA, USA) for western blot analysis. The PVDF membranes were incubated with a primary monoclonal anti-SETD1B antibody (cat. no. ab113984; Abcam, Cambridge, MA, USA; 1:500) at $2-8^{\circ} \mathrm{C}$ overnight and were subsequently incubated with a horseradish peroxidase (HRP)-conjugated secondary antibody (cat. no. RABHRP1; Sigma-Aldrich; Merck KGaA, Darmstadt, Germany; 1:1,000) for $1 \mathrm{~h}$ at room temperature. Targeted SETD1B protein bands were visualized using an enhanced chemiluminescence kit (Pierce; Thermo Fisher Scientific, Inc.). The primary antibody for $\beta$-actin (cat. no. 3700; Cell Signaling Technology, Inc., Danvers, MA, USA; 1:1,000) was used to as a loading control for the western blot analysis.
IHC staining. IHC experiments were conducted as previously described (9). The deparaffinized sections were boiled for $2.5 \mathrm{~min}$ in citrate buffer, $\mathrm{pH}$ 6.0. Endogenous peroxidase activity was blocked through incubation with a $3 \%$ hydrogen peroxide solution for $20 \mathrm{~min}$ at room temperature. Subsequently, the $4-\mu \mathrm{m}$ sections were incubated for $24 \mathrm{~h}$ at $4^{\circ} \mathrm{C}$ with a primary monoclonal anti-SETD1B antibody (Abcam; cat. no. ab113984; 1:500) and subsequently with an HRP-labeled anti-rabbit immunoglobulin G secondary antibody (cat. no. AP101P; Sigma-Aldrich; Merck KGaA; 1:1,000) for $2 \mathrm{~h}$ at $37^{\circ} \mathrm{C}$. The positive cells were analyzed in five distinct fields and images were captured under different magnifications using a light microscope (Olympus Corporation, Tokyo, Japan; magnification, x100 and x200).

Statistical analysis. Data are presented as the mean \pm standard deviation. Statistical analyses were performed using the SPSS 20.0 software (IBM Corp., Armonk, NY, USA). The Wilcoxon test was used to determine SETD1B expression in HCC paired tissues. A paired t-test was used to compare SETD1B expression in RNA-Seq studies.

RNA-Seq data of 66 paired HCC samples were retrieved from The Cancer Genome Atlas (TCGA) Firehose pipeline on the Broad Institute website (http://gdac.broadinstitute.org/). Kaplan-Meier curves were conducted to analyze the associations between the SETD1B expression levels and the overall survival (OS) or disease-free survival (DFS) rates of the patients with HCC. Univariate and multivariate cox regression analysis were performed with the Cox proportional hazards regression model to determine the effects of prognostic factors on survival. $\mathrm{P}<0.05$ was considered to indicate a statistically significant difference.

\section{Results}

SETD1B is upregulated in HCC samples and cell lines. To investigate the role of SETD1B in the development and progression of HCC, RT-qPCR was used to examine the SETD1B expression levels in 76 pairs of HCC tissues and adjacent nontumor tissue samples. As observed in Fig. 1, SETD1B levels were significantly upregulated in the HCC tissues compared with those in the adjacent normal tissues $(\mathrm{P}<0.001$; Fig. 1A-B). To additionally confirm the overexpression of SETD1B in HCC tissues, 4 pairs of HCC and adjacent normal tissues were selected to evaluate the protein levels of SETD1B by western blot analysis. As expected, it was visually observed that the western blot analysis results were consistent with the RT-qPCR data (Fig. 1C). The expression levels of SETD1B in 2 human HCC cell lines (97L and HCCLM3) were also determined and upregulated SETD1B expression levels were observed in the HCC cell lines compared with that in LO2 cells (Fig. 1D). In addition, the SETD1B expression levels of 66 paired HCC samples in The Cancer Genome Atlas (TCGA) RNA-Seq data set were analyzed, and it was identified that SETD1B levels were also significantly increased in these samples (Fig. 1E). These results prompted additional investigation into the potential role of SETD1B in HCC carcinogenesis.

Upregulation of SETDIB in HCC tissues determined by $I H C$. Next, to additionally clarify whether SETD1B was upregulated at the protein level, IHC was performed to 
A

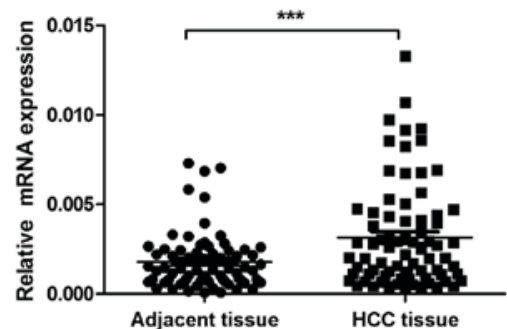

C $\begin{array}{llllllll}\text { T1 } & \text { N1 } & \text { T2 } & \text { N2 } & \text { T3 } & \text { N3 } & \text { T4 } & \text { N4 }\end{array}$ $\longrightarrow \cdots \cdots-$

$\beta$-actin

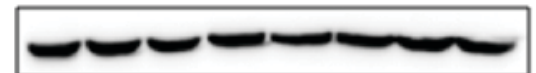

B

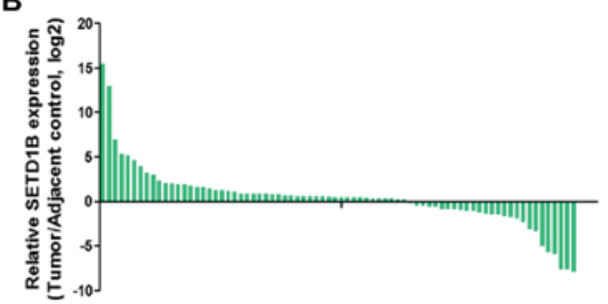

E

TCGA RNA-Seq

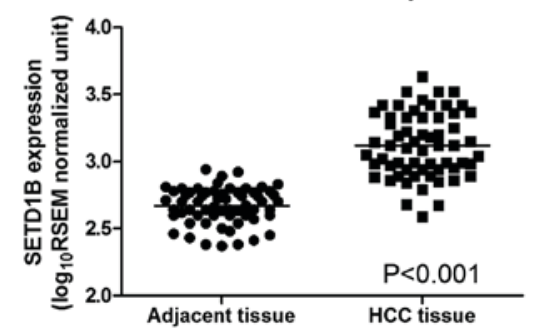

Figure 1. SETD1B overexpression in HCC samples examined by RT-qPCR and western blot analysis. (A) The mRNA levels of SETD1B in 76 HCC tissues and paired adjacent normal tissues were detected by RT-qPCR. ${ }^{* * *} \mathrm{P}<0.001$ determined by Wilcoxon test. (B) Positive values indicate an increased level of SETD1B expression in the tumor tissue compared with adjacent normal tissues, while negative values indicate a decreased level of SETD1B expression in the tumor tissue compared with that in adjacent normal tissues. (C) The SETD1B protein levels in the tissues of 4 patients with HCC were determined by western blot analysis. B-actin was used as a control. (D) The SETD1B protein levels in the HCC 97L and HCCLM3 cell lines were determined by western blot analysis. (E) The upregulation of SETD1B was observed in 66 pairs of HCC samples from TCGA database. Horizontal lines represent the median expression levels. SETD1B, SET domain-containing 1B; HCC, hepatocellular carcinoma; RT-qPCR, reverse transcription quantitative polymerase chain reaction; TCGA, The Cancer Genome Atlas; RSEM, RNA-seq by expectation maximization.

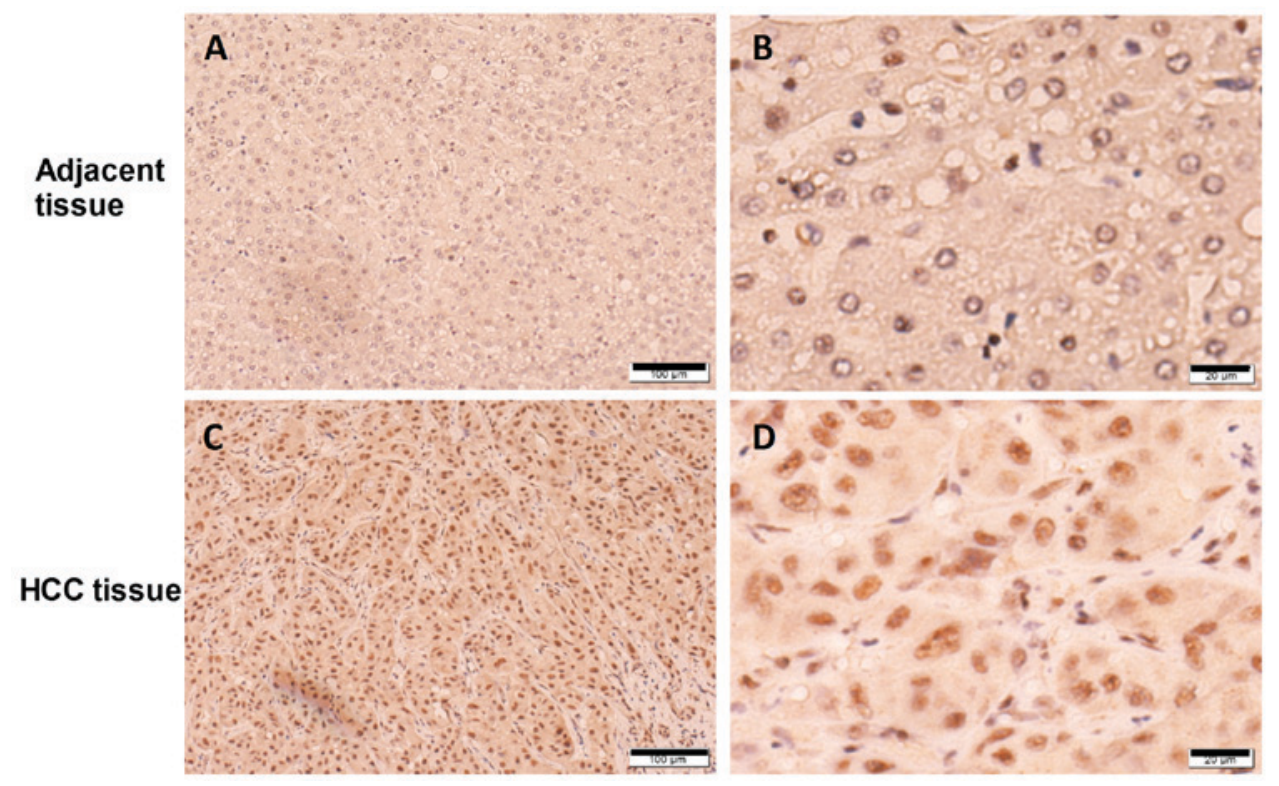

Figure 2. SETD1B expression in HCC tissues based on immunohistochemical staining. Representative images demonstrate the upregulation of the SETD1B protein in HCC tissues compared with that in adjacent normal tissues. (A and B) Negative SETD1B staining in the normal tissue adjacent to HCC at (A) magnification, x100 and (B) magnification, x200. (C and D) High SETD1B expression levels in the HCC tissue at (C) magnification, x100 and (D) magnification, x200. SETD1B, SET domain-containing 1B; HCC, hepatocellular carcinoma.

examine SETD1B expression in sections of paired HCC tissue samples. It was visually observed that SETD1B expression was primarily located in the nucleus and was markedly increased in the HCC cells compared to that in 

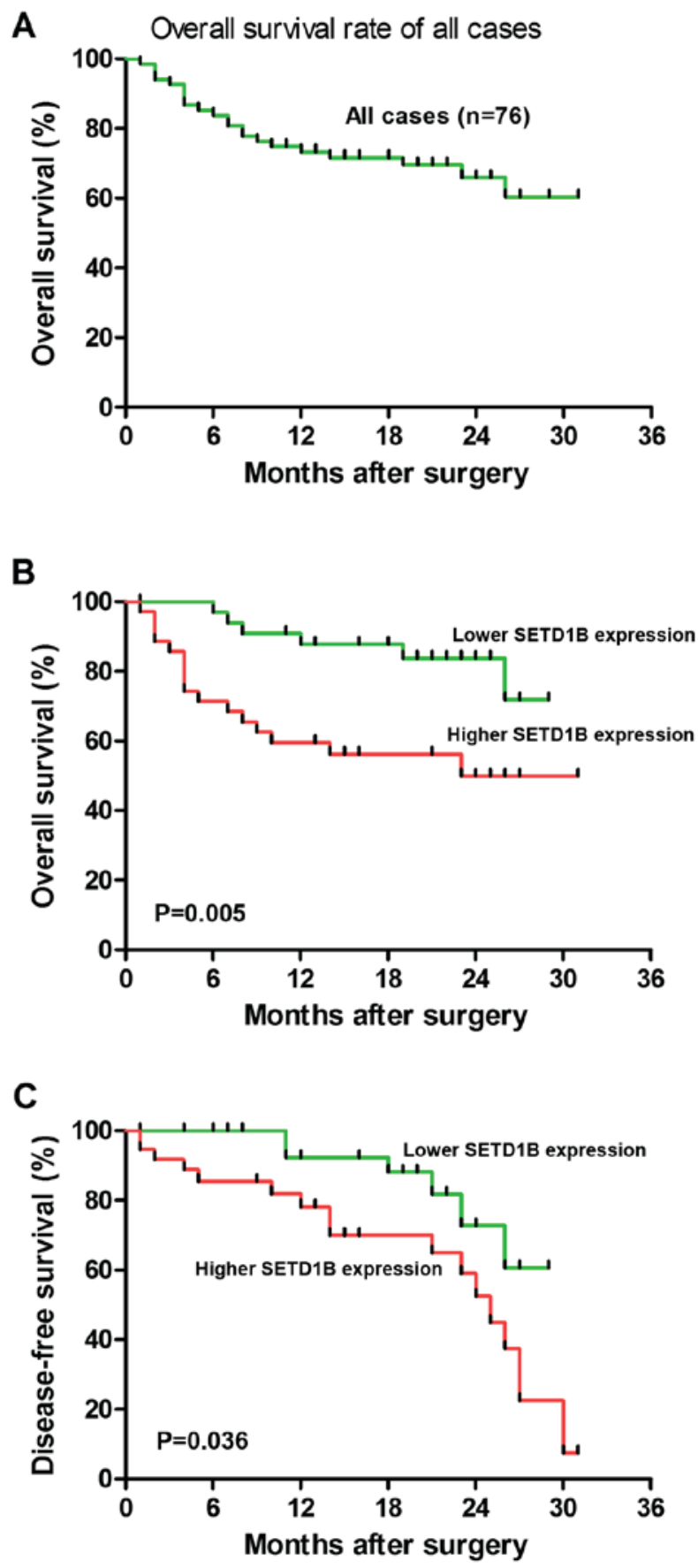

Figure 3. Association between SETD1B expression and HCC patient survival rates as indicated by Kaplan-Meier analysis. (A) The three-year overall survival rate of $76 \mathrm{HCC}$ patients was $60 \%$. (B) Patients with HCC with decreased SETD1B expression levels exhibited significantly longer overall survival times compared with those with increased levels of SETD1B expression ( $\mathrm{P}=0.005$, log-rank test). (C) Patients with HCC with decreased SETD1B expression levels exhibited significantly longer disease-free survival times compared with those with higher SETD1B expression levels $(\mathrm{P}=0.036$, log-rank test). SETD1B, SET domain-containing 1B; HCC, hepatocellular carcinoma.

normal adjacent hepatocytes (Fig. 2). Taking together, it was concluded that SETD1B was upregulated in human HCC tissues at the mRNA and protein levels.

Association between SETDIB protein expression and the clinicopathological features of HCC. To additionally explore whether SETD1B expression in HCC tissues determined the clinical prognosis in patients with $\mathrm{HCC}, 76$ patients with $\mathrm{HCC}$ were divided into high SETD1B and low SETD1B expression groups according to the mean value of the expression levels of SETD1B in HCC samples. As demonstrated in Table I, it was identified that high SETD1B expression was closely associated with tumor size $(\mathrm{P}<0.05)$, clinical tumor stage $(\mathrm{P}<0.01)$ and whether or not liver cirrhosis was present $(\mathrm{P}<0.05)$. By contrast, no association was observed between SETD1B expression and other parameters, including sex, age, the $\alpha$-fetoprotein level, smoking status, drinking status, recurrence and portal vein tumor thrombus (PVTT) $(\mathrm{P}>0.05)$.

SETDIB expression and HCC patient survival. To additionally evaluate whether SETD1B expression exhibited prognostic potential for the OS of patients with $\mathrm{HCC}$, the association between SETD1B expression and HCC patient survival rates was analyzed using Kaplan-Meier analyses. As indicated in Fig. 3, the 3-year OS rate of the 76 patients with HCC was $60 \%$. In addition, the associations between SETD1B expression and the survival outcomes of the patients with HCC were investigated using on Kaplan-Meier analyses. The results suggested that an increased SETD1B expression level in HCC tissues was significantly associated with a decrease in $\mathrm{OS}(\mathrm{P}=0.005$; Fig. 3B) and DFS ( $\mathrm{P}=0.036$; Fig. 3C) during the 3 -year follow-up period. In addition, survival benefits were observed in patients with a small tumor size $(\mathrm{P}=0.003)$, with an early clinical stage $(\mathrm{P}=0.041)$, without PVTT $(\mathrm{P}=0.043)$ and without liver cirrhosis $(\mathrm{P}=0.021)$. Multivariate Cox regression analysis revealed that SETD1B expression [relative risk $(\mathrm{RR})=4.151 ; \mathrm{P}=0.016]$, tumor size $(\mathrm{RR}=8.639 ; \mathrm{P}=0.001)$ and clinical stage $(\mathrm{RR}=6.371 ; \mathrm{P}=0.006)$ were independent prognostic markers for OS in patients with HCC (Table II), indicating that SETD1B is essential for the development, progression and outcomes of $\mathrm{HCC}$.

\section{Discussion}

To the best of our knowledge, the present study demonstrated for the first time that SETD1B expression is significantly increased in HCC tissues compared with that in adjacent normal tissues. Specifically, the increased expression of SETD1B in HCC was associated with tumor size, a more advanced clinical stage and the development of liver cirrhosis. In addition, it was identified that increased SETD1B expression was associated with decreased OS rates. Furthermore, Cox regression analysis demonstrated that SETD1B is an independent predictive marker for the prognosis of HCC. This result was additionally validated with a larger cohort of HCC samples from the TCGA database. These data suggested that SETD1B may serve critical roles in HCC development and progression, and monitoring SETD1B levels may have potential clinical applications. However, insights into the mechanism of how overexpression, not mutation, contributes to a poor outcome in $\mathrm{HCC}$ require additional investigation.

SETD1B encodes a histone H3 Lysine 4 (H3K4)-met hyltransferase and is a component of the SET1 complex (SETIC)/complex proteins associated with Set1 complex, which participates in a number of biological processes (10-13). For example, a previous study demonstrated that tumor cells use 
Table I. Association between SETD1B expression and clinicopathological features in hepatocellular carcinoma.

\begin{tabular}{|c|c|c|c|c|}
\hline \multirow[b]{2}{*}{ Parameters } & \multirow[b]{2}{*}{ No. of patients } & \multicolumn{2}{|c|}{ SETD1B expression } & \multirow[b]{2}{*}{ P-value } \\
\hline & & Low & High & \\
\hline \multicolumn{5}{|l|}{ Age, years } \\
\hline$<60$ & 58 & 31 & 27 & \multirow[t]{2}{*}{0.280} \\
\hline$\geq 60$ & 18 & 7 & 11 & \\
\hline \multicolumn{5}{|l|}{ Sex } \\
\hline Male & 68 & 33 & 35 & \multirow[t]{2}{*}{0.455} \\
\hline Female & 8 & 5 & 3 & \\
\hline \multicolumn{5}{|l|}{ Tumor size, $\mathrm{cm}$} \\
\hline$<5$ & 48 & 28 & 20 & \multirow[t]{2}{*}{$0.028^{\mathrm{a}}$} \\
\hline$\geq 5$ & 28 & 9 & 19 & \\
\hline \multicolumn{5}{|l|}{ AFP } \\
\hline$<20$ & 24 & 13 & 11 & \multirow[t]{2}{*}{0.622} \\
\hline$\geq 20$ & 52 & 25 & 27 & \\
\hline \multicolumn{5}{|c|}{ Histological grade } \\
\hline Well/moderate & 70 & 34 & 36 & \multirow[t]{2}{*}{0.395} \\
\hline Poor & 6 & 4 & 2 & \\
\hline \multicolumn{5}{|l|}{ Clinical stage } \\
\hline $\mathrm{I}-\mathrm{II}$ & 59 & 39 & 20 & \multirow[t]{2}{*}{$0.007^{\mathrm{b}}$} \\
\hline III-IV & 17 & 5 & 12 & \\
\hline \multicolumn{5}{|c|}{ Number of tumors } \\
\hline Single & 63 & 30 & 33 & \multirow[t]{2}{*}{0.361} \\
\hline Multiple & 13 & 8 & 5 & \\
\hline \multicolumn{5}{|c|}{ Alcohol consumption } \\
\hline Yes & 32 & 18 & 14 & \multirow[t]{2}{*}{0.353} \\
\hline No & 44 & 20 & 24 & \\
\hline \multicolumn{5}{|l|}{ Smoking status } \\
\hline Yes & 42 & 20 & 22 & \multirow[t]{2}{*}{0.645} \\
\hline No & 34 & 18 & 16 & \\
\hline \multicolumn{5}{|l|}{ Recurrence } \\
\hline Yes & 23 & 12 & 11 & \multirow[t]{2}{*}{0.803} \\
\hline No & 53 & 26 & 27 & \\
\hline \multicolumn{5}{|c|}{ Portal vein tumor thrombus } \\
\hline Yes & 42 & 21 & 21 & \multirow[t]{2}{*}{0.896} \\
\hline No & 34 & 17 & 17 & \\
\hline Microvascular in & & & & \\
\hline Yes & 61 & 32 & 29 & 0.387 \\
\hline No & 15 & 6 & 9 & \\
\hline Liver cirrhosis & & & & \\
\hline Absent & 27 & 16 & 11 & $0.039^{\mathrm{a}}$ \\
\hline Present & 49 & 17 & 32 & \\
\hline
\end{tabular}

Results are expressed as the mean of $2^{-\Delta \Delta \mathrm{Cq}}$ values. ${ }^{\mathrm{a}} \mathrm{P}<0.05 ;{ }^{\mathrm{b}} \mathrm{P}<0.01$. SETD1B, SET domain-containing $1 \mathrm{~B}$.

the SETD1B-Histone H3 lysine 4 trimethylation (H3K4me3) epigenetic axis to bypass the normal role of interferon regulatory factor 8 expression in activating inducible nitric oxide synthase (iNOS) expression in myeloid-derived suppressor cells under pathological conditions (14). Setd1b deficiency causes female sterility in mice and serves as a maternal effect gene by regulating the oocyte gene expression program (15). The MLL/Setd1b methyltransferase is required for Spemann's organizer gene activation in Xenopus (16). A frameshift mutation in the histone methylation-associated gene SETD1B results in its regional heterogeneity in gastric and colorectal cancer with high microsatellite instability (17). Song et al (18) 
Table II. Univariate and multivariate cox regression analysis for overall survival in 76 patients with HCC.

\begin{tabular}{|c|c|c|c|c|}
\hline \multirow[b]{2}{*}{ Variable } & \multicolumn{2}{|c|}{ Univariate regression analysis } & \multicolumn{2}{|c|}{ Multivariate regression analysis } \\
\hline & RR & P-value & $\mathrm{RR}$ & P-value \\
\hline \multicolumn{5}{|l|}{ Age } \\
\hline$<60$ & 1.453 & & 1 & \\
\hline$\geq 60$ & & 0.456 & 1.296 & 0.625 \\
\hline \multicolumn{5}{|l|}{ Sex } \\
\hline Male & 0.981 & & 1 & \\
\hline Female & & 0.956 & 1.032 & 0.968 \\
\hline Tumor size (cm) & 1 & & & \\
\hline$<5 \mathrm{~cm}$ & 6.138 & & 1 & \\
\hline$\geq 5 \mathrm{~cm}$ & & 0.003 & 8.639 & 0.001 \\
\hline$\alpha$-fetoprotein & 1 & & & \\
\hline$<20$ & 0.738 & & 1 & \\
\hline$\geq 20$ & & 0.536 & 0.683 & 0.428 \\
\hline Histological grade & 1 & & & \\
\hline Well/moderate & 1.956 & & 1 & \\
\hline Poor & & 0.396 & 4.175 & 0.096 \\
\hline Clinical stage & 1 & & & \\
\hline I-II & 5.42 & & & \\
\hline III-IV & & 0.041 & 6.371 & 0.006 \\
\hline Number of tumors & 1 & & & \\
\hline Single & 8.624 & & 1 & \\
\hline Multiple & & 0.361 & 6.751 & 0.132 \\
\hline Alcohol consumption & 1 & & & \\
\hline Yes & 1.435 & & 1 & \\
\hline No & & 0.416 & 1.369 & 0.652 \\
\hline Smoking status & 1 & & & \\
\hline Yes & 1.466 & & 1 & \\
\hline No & & 0.465 & 1.096 & 0.725 \\
\hline Recurrence & 1 & & & \\
\hline Yes & 1.853 & & 1 & \\
\hline No & & 0.656 & 1.496 & 0.854 \\
\hline Portal vein tumor thrombus & 1 & & & \\
\hline Yes & 1.493 & & & \\
\hline No & & 0.043 & & \\
\hline Microvascular invasion & 1 & & & \\
\hline Yes & 6.453 & & 1 & \\
\hline No & & 2.456 & 4.296 & 0.625 \\
\hline Liver cirrhosis & 1 & & & \\
\hline Absent & 5.62 & & & \\
\hline Present & & 0.021 & & \\
\hline SETD1B expression & 1 & & & \\
\hline Low & 5.123 & & 1 & \\
\hline High & & & & \\
\hline
\end{tabular}

RR, relative risk; SETD1B, SET domain-containing 1B.

identified that SETD1B, as one of the important histone regulator genes, is frequently altered in esophageal squamous cell carcinoma. The molecular mechanisms for SETDIB function in carcinogenesis and cancer progression have not been 
clearly elucidated. Although a role of SETD1B as an oncogene in $\mathrm{HCC}$ has been suggested in the present study, the underlying mechanisms of increased SETD1B expression in HCC progression remain largely elusive. For example, to verify that the significance of SETD1B in HCC is based on epigenetic modification, subsequent studies with chromatin immunoprecipitation PCR in HCC tissues are required to assess whether the H3K3 modifications are enhanced around the critical transcription factor genes that have been suggested to significantly contribute to $\mathrm{HCC}$ pathogenesis. In addition, a limited number of HCC tissue samples were analyzed in the present study, and SETD1B expression levels require confirmation with larger cohorts of HCC clinical samples. Whether SETD1B may also be detected in the plasma or even in circulating exosomes and whether the circulating SETD1B is also associated with HCC development are also key issues to consider. Therefore, the role of SETD1B in HCC progression requires investigation, and will be a focus in subsequent studies.

SETD1B catalyzes the methylation of $\mathrm{H} 3 \mathrm{~K} 4$, and several other enzymes including SETD1A, histone-lysine N-methyltransferase SETD7, MLL1-4 and histone-lysine N-methyltransferase SMYD (SMYD) 1-3 catalyze the same reaction (11). The level of $\mathrm{H} 3 \mathrm{~K} 4$ methylation affects gene transcription, but this level depends not only on the activity of these methyltransferases but also on that of demethylases, or 'erasers' (19). In humans, the erasers for H3K4me are lysine-specific histone demethylase (LSD)1-2, lysine-specific demethylase 5A-D and ribosomal oxygenase 1. For example, a previous study described the role of the SMYD3 histone methyltransferase in tumorigenesis and whether the effects are local or global (19). JARID1B promotes metastasis and the epithelial-mesenchymal transition via phosphatase and tensin homolog/protein kinase B signaling in HCC cells (20). While it is true that the role of SETD1B in HCC has not been described at present, those other 'writers' or 'erasers' also affect the level of the same epigenetic marker, H3K4me3, that results from SETD1B action. It has been suggested that a high level of JARID1B expression was associated with decreased OS in patients with HCC (20). Considering that SETD1B and JARID1B induce opposite effects on the level of H3K4me3, it was hypothesized that the reason for these results is a small sample size and individual differences in the patients with HCC.

In summary, the results of the present study reveal that SETD1B expression is markedly upregulated in HCC tissues, and is associated with a poor prognosis in patients with HCC. The overexpression of SETD1B was associated with tumor size, clinical stage and the presence of liver cirrhosis. These data demonstrate that SETD1B has potential as a predictive marker for prognosis and as a therapeutic drug target for $\mathrm{HCC}$.

\section{Acknowledgements}

Not applicable.

\section{Funding}

The present study was supported by a grant from the National Natural Science Foundation of China (grant no. 81601860).

\section{Availability of data and materials}

All data generated or analyzed during this study are included in this published article.

\section{Authors' contributions}

DC, CW, GL, RW, ZW and LY performed the experiments. DC, TL, PZ and JY gathered clinical samples and performed the clinical analysis. XW, SZ and PY designed the study and wrote the manuscript. All authors read and approved the final manuscript.

\section{Ethics approval and consent to participate}

Informed consent was obtained from the enrolled patients. The present study was approved by the Ethics Committee of Beijing 302 Hospital (Beijing, China).

\section{Patient consent for publication}

Written informed consent was obtained from the enrolled patients.

\section{Competing interests}

The authors declare that they have no competing interests.

\section{References}

1. Yang Y, Zhou Y, Hou J, Bai C, Li Z, Fan J, Ng IOL, Zhou W, Sun H, Dong Q, et al: Hepatic IFIT3 predicts interferon- $\alpha$ therapeutic response in patients of hepatocellular carcinoma. Hepatology 66: 152-166, 2017.

2. El-Serag HB: Hepatocellular carcinoma. N Engl J Med 365: 1118-1127, 2011.

3. Cheng AL, Kang YK, Lin DY, Park JW, Kudo M, Qin S, Chung HC, Song X, Xu J, Poggi G, et al: Sunitinib versus sorafenib in advanced hepatocellular cancer: Results of a randomized phase III trial. J Clin Oncol 31: 4067-4075, 2013.

4. Lee JH, Tate CM, You JS and Skalnik DG: Identification and characterization of the human Set1B histone H3-Lys4 methyltransferase complex. J Biol Chem 282: 13419-13428, 2007.

5. Duncan EM, Chitsazan AD, Seidel CW and Alvarado AS: Set1 and MLL1/2 target distinct sets of functionally different genomic loci in vivo. Cell Rep 17: 930, 2016.

6. Li Y, Han J, Zhang Y, Cao F, Liu Z, Li S, Wu J, Hu C, Wang Y, Shuai J, et al: Structural basis for activity regulation of MLL family methyltransferases. Nature 530: 447-452, 2016.

7. Zhang BL, Ji X, Yu LX, Gao Y, Xiao CH, Liu J, Zhao DX, Le Y, Diao GH, Sun JY, et al: Somatic mutation profiling of liver and biliary cancer by targeted next generation sequencing. Oncol Lett 16: 6003-6012, 2018.

8. Livak KJ and Schmittgen TD: Analysis of relative gene expression data using real-time quantitative PCR and the 2(-Delta Delta C(T)) method. Methods 25: 402-408, 2001.

9. Li J, Wu H, Li W, Yin L, Guo S, Xu X, Ouyang Y, Zhao Z, Liu S, Tian Y, et al: Downregulated miR-506 expression facilitates pancreatic cancer progression and chemoresistance via SPHK1/Akt/NF- $\mathrm{KB}$ signaling. Oncogene 35: 5501-5514, 2016.

10. Davie JR, Xu W and Delcuve GP: Histone H3K4 trimethylation: Dynamic interplay with pre-mRNA splicing. Biochem Cell Biol 94: 1-11, 2016.

11. Yang W and Ernst P: Distinct functions of histone H3, lysine 4 methyltransferases in normal and malignant hematopoiesis. Curr Opin Hematol 24: 322-328, 2017.

12. Hiraide T, Nakashima M, Yamoto K, Fukuda T, Kato M, Ikeda H, Sugie Y, Aoto K, Kaname T, Nakabayashi K, et al: De novo variants in SETD1B are associated with intellectual disability, epilepsy and autism. Hum Genet 137: 95-104, 2018. 
13. Schmidt K, Zhang Q, Tasdogan A, Petzold A, Dahl A, Arneth BM, Slany R, Fehling HJ, Kranz A, Stewart AF and Anastassiadis K: The H3K4 methyltransferase Setdlb is essential for hematopoietic stem and progenitor cell homeostasis in mice. Elife 7: e27157, 2018

14. Redd PS, Ibrahim ML, Klement JD, Sharman SK, Paschall AV Yang D, Nayak-Kapoor A and Liu K: SETD1B activates iNOS expression in myeloid-derived suppressor cells. Cancer Res 77: 2834-2843, 2017

15. Brici D, Zhang Q, Reinhardt S, Dahl A, Hartmann H, Schmidt K, Goveas N, Huang J, Gahurova L, Kelsey G, et al: Setd1b, encoding a histone 3 lysine 4 methyltransferase, is a maternal effect gene required for the oogenic gene expression program. Development 144: 2606-2617, 2017.

16. Lin H, Min Z and Tao Q: The MLL/Setdlb methyltransferase is required for the Spemann's organizer gene activation in Xenopus. Mech Dev 142: 1-9, 2016.

17. Choi YJ, Oh HR, Choi MR, Gwak M, An CH, Chung YJ, Yoo NJ and Lee SH: Frameshift mutation of a histone methylation-related gene SETD1B and its regional heterogeneity in gastric and colorectal cancers with high microsatellite instability. Hum Pathol 45: 1674-1681, 2014.
18. Song Y, Li L, Ou Y, Gao Z, Li E, Li X, Zhang W, Wang J, Xu L, Zhou Y, et al: Identification of genomic alterations in oesophageal squamous cell cancer. Nature 509: 91-95, 2014.

19. Medjkane S, Cock-Rada A and Weitzman JB: Role of the SMYD3 histone methyltransferase in tumorigenesis: Local or global effects? Cell Cycle 11: 1865, 2012.

20. Tang B, Qi G, Tang F, Yuan S, Wang Z, Liang X, Li B, Yu S, Liu J, Huang Q, et al: JARID1B promotes metastasis and epithelial-mesenchymal transition via PTEN/AKT signaling in hepatocellular carcinoma cells. Oncotarget 6: 12723-12739, 2015 .

This work is licensed under a Creative Commons Attribution-NonCommercial-NoDerivatives 4.0 International (CC BY-NC-ND 4.0) License. 